\title{
Bestimmung des Acetons mit Vanillin und mit Salicylaldehyd
}

\author{
Von F. BAHNer und G. Schulze \\ Aus der Abteilung fïr Endokrinologie der Mediqinischen Universitäts-Poliklinik Heidelberg \\ (Leiter: Prof. Dr. F. Babner)
}

(Der Schriftleitung zugegangen am 9. Mai 1964)

\begin{abstract}
Aus Aceton und Vanillin entsteht in alkalischer Lösung rotes Divanillalaceton. Durch Variation der Vanillinund KOH-Konzentrationen wird als günstigstes Reagenz zur Acetonbestimmung 0,4\% Vanillin in $5 n-\mathrm{KOH}$ gefunden. Mit einem Zusatz von Methanol löst sich Vanillin in höherer Konzentration. Das Reaktionsprodukt Divanillalaceton in alkalischer Lösung wird durch Tageslicht in Luft- oder $\mathrm{N}_{2}$-Atmosphäre gebleicht. Es wird in methanolhaltiger Lösung in Luftgegenwart durch Licht schnell dunkel. Divanillalaceton in alkalischer Lösung ändert beim Temperaturwechsel langsam und reversibel die Farbtiefe, es wird mit steigender Temperatur heller. In methanolhaltiger alkalischer Lösung unterliegt es außer diesem reversiblen Farbwechsel einer irreversiblen, stetigen Zunahme der Farbtiefe. Die Extinktion des Divanillalacetons hängt von der Reaktionstemperatur ab.

Die Reaktion des Acetons mit Vanillin wird mit der Reaktion des Acetons mit Salicylaldehyd verglichen. Unter günstigsten Bedingungen ist bei gleicher Acetonmenge die Extinktion des Disalicylacetons fast 4mal höher als die des Divanillalacetons. Mit methanolhaltiger alkalischer Vanillinlösung erzielt man etwiva gleich hohe Extinktionen wie mit der Salicylaldehydreaktion, der Methanolzusatz stört die Acetonbestimmung aber erheblich. Vanillin eignet sich zur Acetonbestimmung daher nicht so gut wie Salicylaldehyd.
\end{abstract}

In alkaline solution, acetone and vanillin give divanillalacetone. By varying the concentrations of vanillin and $\mathrm{KOH}$, a suitable reagent for the determination of acetone is found to be $0.4 \%$ vanillin in $5 n \mathrm{KOH}$. In alkaline medium, the reaction product, divanillalacetone, in bleached by daylight in an atmosphere of ait or nitrogen. In a solution containing methanol it darkens rapidly in the light in the presence of air. In alkaline solution, the colour intensity of divanillalacetone changes slowly and reversibly with changes in temperature; it becomes lighter with increasing temperature. In an alkaline solution containing methanol, this reversible colour change is superimposed on an irreversible, continual increase in the intensity of the colour. The extinction of divanillalacetone depends on the reaction temperature.

The reaction of acetone with vanillin is compared with the reaction of acetone with salicylaldehyde. Under optimum conditions, at equal concentrations of acetone, the extinction of disalicylacetone is almost four times higher than that of divanillalacetone. In an alkaline solution of vanillin containing methanol, the extinction is about the same as with the salicylaldehyde reaction, but the methanol inte1feres greatly in the acetone determination. Vanillin is thus not as suitable as salicylaldehyde for the determination of acetone.

Aceton verbindet sich mit Vanillin in alkalischer Lösung zu rotem Divanillalaceton (I), mit Salicylaldehyd zu rotem Disalicylaceton (II):

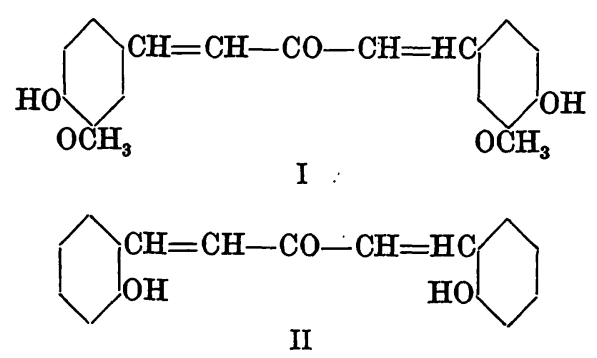

Beide Reaktionen sind zur quantitativen Bestimmung des Acetons benutzt worden. Nikitrin (1), BäCKström (2) und Levine und Taterka (3) sehen einen Vorteil des Vanillins darin, daß es bei Zimmertemperatur kristallisiert und daher genauer wägbar ist; es ist beständiger, besser wasserlöslich als Salicylaldehyd, und. seine Lösungen verfärben sịch bei längerem Stehen viel weniger als die des Salicylaldehyds. - Die Reaktion des Acetons mit Salicylaldehyd ist von BAHNER (4) eingehend untersucht worden. Er fand für die quantitative Bestimmung des Acetons folgende wichtige Reaktionsbedingungen:

1. Eine alkalische Salicylaldehydlösung wird an der Luft, nicht aber unter Stickstoff dunkel.
2. Dicse Sauerstoffempfindlichkeit ist lichtabhängig, sic ist bei Tageslicht groß, im Dunkeln und bei künstlichem Licht aber gering.

3. Die alkalische Salicylaldehydlösung verändert beim Temperaturwechsel reversibel ihre Farbtiefe, sie wird mit steigender Temperatur dunkler.

4. Eine alkalische Disalicylacetonlösung verändert mit der Temperatur langsam und reversibel die Farbtiefe, sie wird mit steigender Temperatur heller.

Arbeitet man nach BAHNER (4) bei Luftabschluß in $\mathrm{N}_{2}$-Atmosphäre und bei künstlicher Beleuchtung und berücksichtigt man die Temperatur während der Photometrie und deren Zeitabstand von der eigentlichen Farbentwicklung, dann gelingt es, mit der Salicylaldehydreaktion sehr genau reproduzierbare Ergebnisse zu erzielen. $0,1 \mu \mathrm{g}\left(\sim 2 \cdot 10^{-9} \mathrm{Mol}\right)$ Aceton in der Probe kann zuverlässig erfaßt werden. Sie erlaubt die Bestimmung der Acetessigsäure in $0,1 \mathrm{~m} l$ Blut (5). Das Aceton wird dabei durch wärmebeschleunigte Mikrodiffusion im Mikrokonvektionsapparat (6) abgetrennt. - Die Licht- und Sauierstoffempfindlichkeit des Salicylaldehyds in alkalischer Lösung beruht auf der phenolischenen Hydroxylgruppe, sie ist daher für das Vanillin ebenfalls zu erwarten. Bei der Ähnlichkeit der Strukturformeln von Disalicylaceton und von Divanillalaceton ist bei diesem auch mit der reversiblen thermischen Farbänderung zu rechnen. 


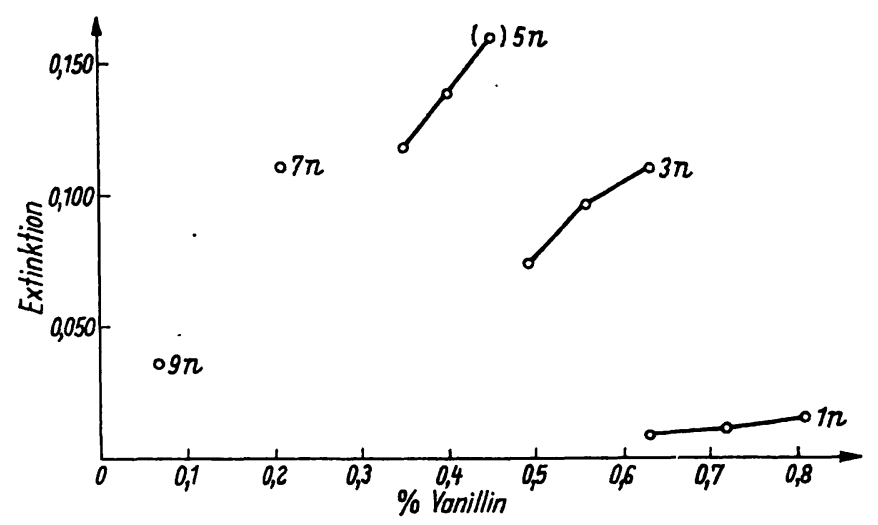

Abb. 1 -

Einfluß der Vanillin- und KOH-Konzentration auf die Extinktion von Divanillalaceton. Acetonmenge konstant $12,5 \mu \mathrm{g}$ in der Probe

Wir beschreiben in dieser Arbeit Versuche über die Reaktion des Acetons mit Vanillin, die zeigen, daß die Vanillinreaktion keine Vorteile gegenüber der Salicylaldehydreaktion hat. LINDBERG und ÖrSTröm (7) haben beide Reaktionen schon einmal verglichen und fanden die Vanillinreaktion besonders bei sehr niedrigen Extinktionen von Vorteil. Damals waren aber die von uns für die Vanillinreaktion und die von BAHNER für die Salicylaldehydreaktion gefundenen Reaktionsumstände unbekannt.

\section{Methodik}

Die Einzelheiten der Methode sind von BAHNER $(4,5)$ ausführlich beschrieben worden. Entweder werden die Reaktionsteilnehmer in ausgezogene Reagenzgläser (oder Ampullen) eingeschmolzen, oder es wird Aceton im Mikrokonvektionsverfahren (6) aus einer Acetessigsäurelösung abgetrennt und mit Vanillin an Stelle von Salicylaldehyd bestimmt. Die Extinktion wird im Photometer ,Eppendorf" bei $492 \mathrm{~m} \mu$ in $1 \mathrm{~cm}$-Küvetten gegen Aqua dest. abgelesen.

\section{Ergebnisse}

Einfluß der Konqentrationen von Vanillin und $K O H$ in wäßriger Lösung

Durch Mischen von 10-n KOH mit 0,7 bis 0,9-proz. wäßr. Vanillinlösungen werden Lösungen hergestellt, die 1 bis $9-n \mathrm{KOH}$ und 0,07 bis $0,81 \%$ Vanillin enthalten. Je $5 \mathrm{ml}$ werden unter $\mathrm{N}_{2}$ in Ampullen oder ausgezogene Reagenzgläser eingefüllt, danñ tverden je $12,5 \mu \mathrm{g}$ Aceton in $0,1 \mathrm{ml}$ einer wäßrigen Acétonlösung oder beim Leerwert $0,1 \mathrm{~m} l$ Wasser zugesetzt, zugeschmolzen und $1 \mathrm{Std}$. bei $37^{\circ}$ erhitzt. Nun wird abgetrocknet und nach 24-stdg. Stehen im Dunklen photometriert. Die Abbildung 1 zeigt die Extinktionen des entstandenen Divanillalacetons als Differenz zwischen der Extinktion des Versuchsansatzes und des Leerwertes.

Man erkennt, daß bei steigender $\mathrm{KOH}-\mathrm{Konzentration}$ die Extinktionen wesentlich höher sind, und daß steigende Vanillinkonzentrationen bei hoher KOH-Konzentration einèn viel stärkeren Farbanstieg ergeben als bei niedriger $\mathrm{KOH}-\mathrm{Konzentration.} \mathrm{Bei} 9-n \mathrm{KOH}$ ist das Vanillin in keiner Konzentration höher als 0,07\% und bei 7-n KOH .nicht höher als 0,21\% löslich. Selbst $0,45 \%$ Vanillin mit $5-n \mathrm{KOH}$ ist nicht stabil (Wert in

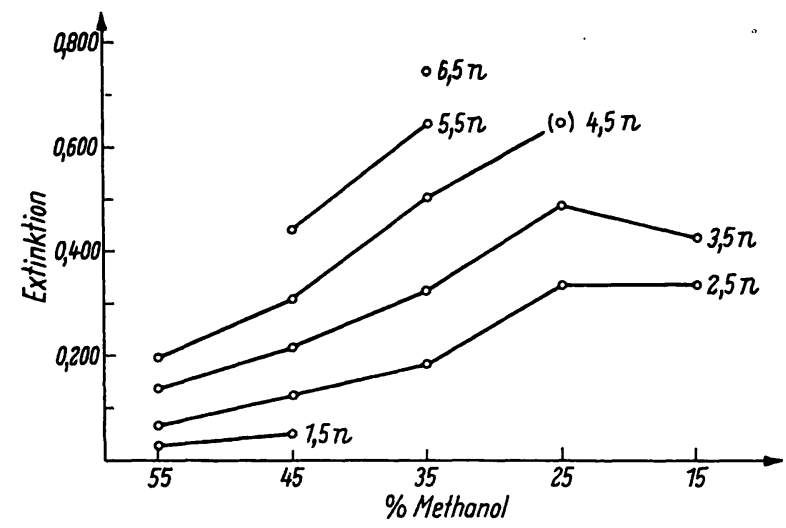

Abb. 2

Einfluß verschiedener Methanol- und $\mathrm{KOH}-\mathrm{Konzentrationen} \mathrm{auf}$ die Extinktion des Divanillalacetons. Im Ansatz konstant 2\% Vanillin und $20,0 \mu \mathrm{g}$ Aceton

Klammern), so daß die höchsten und damit günstigsten Konzentrationen $0,4 \%$ Vanillin in $5-n \mathrm{KOH}$ sind. Hier ist die Extinktion bei 12,5 $\mu \mathrm{g}$ Aceton im Versuch 0,140, während bei einem Ansatz, der eine höhere Vanillinkonzentration unter Zusatz von Methanol enthält (siehe unten), Extinktionen von über 0,5 erreicht werden. Vorteilhaft ist, daß die Extinktionen der Leerwerte bei den Vanillin-KOH-Gemischen ohne Methanolzusatz viel niedriger sind als mit Methanol. Bei den Extinktionen der Abbildung 1 ist der höchste Leerwert 0,021 $(0,81 \%$ Vanillin in $1-n \mathrm{KOH})$ und beim optimalen Gemisch von $0,4 \%$ Vanillin in $5-n \mathrm{KOH}$ ist er nur 0,014 .

Der Nachteil der niedrigen Divanillalaceton-Extinktionen bei schwächerer $\mathrm{KOH}$-Konzentration überwiegt den Vorteil der kleinen Leerwerte. Es ist also vorteilhaft, in stärkerer $\mathrm{KOH} \mathrm{zu}$ arbeiten. Die dabei erreichbaren Extinktionen sind aber wesentlich niedriger als bei der Salicylaldehyd-Methode.

\section{Einfluß der Konzentrationen von Vanillin und $K O H$ bei Methanolzusatz}

Vanillin ist in Methanol besser löslich als in Wasser, so daß verschiedene Autoren (7) zur Acetonbestimmung dem Vanillin Methanol zugesetzt haben.

In mehreren $20 \mathrm{ml}$-Meßkolben werden aus Methanol, Wasser und $10-n \mathrm{KOH}$ unter Zugabe von $1 \mathrm{ml} 40$-proz. Vanillinlösung in Methanol Lösungen hergestellt, die 1,5 bis $6,5-n \mathrm{KOH}, 15$ bis $55 \%$ Methanol und konstant jeweils $2 \%$ Vanillin enthalten. Je $5 \mathrm{ml}$ dieser Mischung werden in die Vorlagen der Mikrokonvektionsapparate eingefüllt und jeweils $20,0 \mu \mathrm{g}$ Acetessigsäure in $0,1 \mathrm{~m} l$ wäßriger Lösung oder beim Leerwert $0,1 \mathrm{~m} l$ Wasser in das Bodengefäß eingefüllt. Die Bestimmung erfolgt in $\mathrm{N}_{2}$-Atmosphäre bei künstlichem Licht, es wird $2 \mathrm{Stdn}$. bei $35^{\circ}$ erhitzt und dann nach 16-stdg. Stehen im Dunkeln photometriert.

Die Abbildung 2 gibt die Extinktionen für das entstandene Divanillalaceton wieder, sie ergeben sich aus der Differenz der Extinktionen der acetonhaltigen Probe zum Leerwert. Man sieht, daß ein Methanolzusatz die Löslichkeit des Vanillins stark erhöht, denn bei einem Methanolgehalt von $55 \%$ ist in 4,5-n KOH $2 \%$ Vanillin löslich, während bei gleicher Alkalinität ohne Methanolzusatz nur etwa 0,5\% Vanillin löslich sind (Abb. 1). Mit steigender $\mathrm{KOH}-$ Konzentration nimmt auch hier 
die Löslichkeit des Vanillins ab, die Farbe des Divanillalacetons aber zu. Steigende Methanol-Konzentrationen schwächen die Farbe ab. Der günstige Effekt kleiner Methanol-Konzentrationen in stärkerem $\mathrm{KOH}-$ Medium wird begrenzt dadurch, daß Vanillin in schwach methanolhaltiger Lösung nicht in der Konzentration von 2\% löslich ist. Erst Lösungen in 3,5-n $\mathrm{KOH}$ bei $15 \%$ Methanolgehalt sind stabil, bei $25 \%$ Methanolgehalt ließ sich die KOH-Konzentration auf 4,5-n steigern, aber in einem zweiten solchen Versuch fiel auch hier Kaliumvanillat aus.

Um die günstigsten Konzentrationen zu ermitteln, muß man bedenken:

1. Die KOH-Konzentration muß möglichst hoch sein. Die oberen Kurven sind günstiger als die unteren.

2. Die Methanol-Konzentrationen müssen möglichst niedrig sein. Der günstigere Kurvenbereich liegt rechts in der Abbildung 2.

Man erkennt, daß die günstigsten Kurvenabschnitte sind: $5,5-n \mathrm{KOH}$ zwischen $45 \%$ und $35 \%$ Methanol und 4,5-n KOH zwischen $35 \%$ und $25 \%$ Methanol. $Z$ wischen beiden Kurven entscheidet man sich für die letztere, weil bei dieser der zugehörige Leerwert etwas kleiner ist. Außerdem läßt sich mit weniger starken $\mathrm{KOH}-L o ̈ s u n g e n ~ l e i c h t e r$ arbeiten. Als günstigste Konzentrationen werden daher gewählt $2 \%$ Vanillin in 4,5-n $\mathrm{KOH}$ und $30 \%$ Methanol.

Bei höheren KOH-Konzentrationen wird der Leerwert relativ hoch. Bei höherer Methanol-Konzentration wird die Endfarbe heller und bei kleiner Methanol-Konzentration sind 2\% Vanillin nicht mehr sicher löslich. Es ist kein Vorteil, die Vanillin-Konzentration über 2\% zu erhöhen, denn höhere Vanillin-Konzentrationen er$z$ wingen einen größeren Methanolzusatz oder eine kleinere $\mathrm{KOH}-\mathrm{Konzentration}$ und beides vermindert die Endfarbe.

\section{Einfluß von Licht und Luft}

Eine Lösung von $2 \%$ Vanillin in $4,5-n \mathrm{KOH}$ unter Zusatz von 30\% Methanol zeigt im Dunkeln folgende Extinktionen:

Sofort nach der Herstellung

0,007

Nach 2 Stdn. (Zimmertemperatur) unter $\mathrm{N}_{2}$

Nach 2 Stdn. (Zimmertemperatur) unter Luft

0,052

0,075 .

Bei Luftzutritt ist die Eigenfarbe der Reagenzlösung nach 2 Stunden also dunkler als unter Stickstoffatmosphäre.

Mikrokonvektionsapparate werden mit $5 \mathrm{ml}$ einer Lösung von $0,4 \%$ Vanillin in 4,5- $n \mathrm{KOH}$ als Vorlage und mit $0,2 \mathrm{ml}$ einer Acetessigsäurelösung, enthaltend 20,0 $\mu \mathrm{g}$ Acetesșigsäure, beschickt. Zum Vergleich wird der Versuch in $\mathrm{N}_{2}$-Atmosphäre oder bei Luftzutritt und im Dunkeln (schwache künstliche Beleuchtung)

Tab. 1

\begin{tabular}{llcc}
\hline & & Dunkel & Licht \\
\hline $\mathrm{N}_{2}:$ & Gesamt-Extinktion & 0,132 & 0,113 \\
& Leerwert & 0,005 & 0,004 \\
& Differenz & 0,127 & 0,109 \\
Luft: & Gesamt-Extinktion & 0,129 & 0,114 \\
& Leerwert & 0,009 & 0,007 \\
& Differenz & 0,120 & 0,107 \\
\hline
\end{tabular}

oder im Tageslicht angestellt. Es wird 1,5 Stdn. im siedenden Wasserbad exhitzt mit der Kühlkappe bei $35^{\circ}$. Nach 20-stdg. Stehen im Dunkeln resp. bei Tageslicht wird photometriert. Das Ergebnis findet sich in Tabelle 1:

Divanillalacetonlösungen ohne Methanolzusatz werden im Licht sowohl bei Luftgegenwart wie bei Luftausschluß heller. Der Einfluß des Lichts ist deutlich, der Unterschied zwischen $\mathrm{N}_{2}$ - und Luftatmosphäre nicht sicher.

Untersucht man in gleicher Weise eine Lösung von $2 \%$ Vanillin in 4,5-n KOH unter Zusatz von 30\% Methanol in der Vorlage, dann ergeben sich die Extinktionen der Tabelle 2. Unter Stickstoffattmosphäre hat Tageslicht

Tab. 2

\begin{tabular}{llcl}
\hline & & Dunkel & L.icht \\
\hline $\mathrm{N}_{2}:$ & Gesamt-Extinktion & 0,777 & 0,777 \\
& Leerwert & 0,109 & 0,112 \\
& Differenz & 0,668 & 0665 \\
Luft: & Gesamt-Extinktion & 0,786 & 1,600 \\
& Leerwert & 0,187 & 0,188 \\
& Differenz & 0,599 & 1,412 \\
\hline
\end{tabular}

also keinen deutlichen Einfluß auf die Farbe des Divanillalacetons, während bei Luftzutritt im gleichen Versuch die Extinktion von 0,599 auf 1,412 ansteigt.

Divanillalaceton in methanolhaltiger Lösung wird im Tageslicht bei Luftzutritt so dunkel, daß Luft- und Lichtgegenwart die quantitative Acetonbestimmung mit Vanillin in methanolhaltigen Lösungen unmöglich machen. Auch das Reagenz, die alkalische methanolhaltige Vanillinlösung wird im Licht bei Luftzutritt dunkler. Schaltet man den Lufteinfluß aus, arbeitet man also in Stickstoffatmosphäre, dann ist die Endfarbe unabhängig von der Beleuchtung, wenn man methanolhaltiges Vanillin verwendet. Schaltet man den Lichteinflu $B$ aus, arbeitet man also im Dunkeln oder bei gedämpftem künstlichem Licht, dann wird bei Luftanwesenheit die Farbe des Divanillalacetons heller, und $z$ war in methanolhaltigen Lösungen deutlicher als in wäßrigen Lösungen. Es ist also wichtiger, die Luft als das Licht bei der Bestimmung auszuschließen. Wäßrige alkalische Vanillinlösungen würden bei Luftgegenwart den kleineren Fehler erzeugen, die erzielten Extinktionen sind aber kleiner als die Extinktionen in methanolhaltigen Lösungen.

\section{Die Farbtiefe in Abbängigkeit von der Zeit}

Es wurden untersucht:

a) $0,4 \%$ Vanillin in 5- $n \mathrm{KOH}$ ohne Methanolzusatz;

b) $2 \%$ Vanillin in 4,5-n $\mathrm{KOH}$ mit 30\% Methanolzusatz; c) als Vergleich $3 \%$ Salicylaldehyd in $3-n \mathrm{KOH}$.

$50 \mathrm{~m} l$ dieser Lösungen werden in Stickstoffatmosphäre und bei künstlicher Beleuchtung in einem Meßkolben mit $0,1 \mathrm{ml}$ einer 0,4-proz. ( $\nabla / v)$ wäßrigen Acetonlösung versetzt, im Wasserbad 1 Std. auf $35^{\circ}$ erwärmt und dann bei Zimmertemperatur stehen gelassen. Sofort nach Abnahme vom Wasserbad $(t=0)$ und nach $1,2 \ldots \ldots 120 \mathrm{Stdn}$. wird je eine Probe photometriert. Ein ebenso angesetzter Meßkolben läuft ohne Acetonzusatz als Leerwert mit $0,1 \mathrm{ml}$ Aqua dest. mit. 
Das in Lösung A „Vanillin ohne Methanol“ entwickelte Divanillalaceton strebt nach dem Temperaturwechsel einem konstanten Farbwert zu, der dann über lange Zeit gleich bleibt. Der Anstieg vom Extinktionswert zur Zeit 0 bis zum Endwert entspricht einer Exponentialfunktion $c=c_{q}\left(1-c^{-k t}\right)$, deren Ablauf eine Halbwertszeit von 2,5 Stdn. hat. Bei der gewählten Acetonmenge nähert sich die Extinktion in $12 \mathrm{Stdn}$. bis auf $2 \%$ dem Endwert.

In Lösung B "Vanillin mit Methanol" wird die Farbe nicht konstant. Die Kurve steigt etwa $20 \mathrm{Stdn}$. lang schnell, dann langsam ständig weiter an. Es ist damit zu rechnen, daß die Geschwindigkeit des Anstiegs von der Temperatur und von der Konzentration der Reaktionsteilnehmer abhängt, und da $\beta$ infolgedessen bei kleinen Schwankungen der Temperatur oder der Konzentrationen ein bestimmter Zeitpunkt, den man wählt um abzulesen, auf einen höher oder niedriger gelegenen Kurvenverlauf fällt, also bei gleicher Acetonmenge eine höhere oder niedrigere Extinktion ergeben kann, was die Reaktion für die quantitative Bestimmung natürlich ungeeignet macht.

Die Farbe des Disalicylacetons, die aus Lösung $C$ entsteht, ändert sich wie die des Divanillalacetons ohne Methanolzusatz und strebt nach dem Temperaturwechsel einem Endwert zu, der dann über viele Stunden konstant ist. Die Endfarbe wird schneller als bei der Vanillinverbindung erreicht, die Halbwertszeit des exponentiellen Farbanstiegs ist 1,0 Stdn. und die Extinktion des Disalicylacetons ist bei der gleichen vorgelegten Acetonmenge bereits nach 3,4 Stdn. bis auf $2 \%$ dem Endwert nahegekommen.

Die Salicylaldehydmethode erweist sich also als vorteilhaft gegenüber der Vanillinmethode ohne Methanolzusatz, weil der Farbwechsel 2,5mal schneller vor sich geht (Halbwertszeit für Disalicylaceton 1 Std., für Divanillalaceton 2,5 Stdn.), und weil die Extinktion fast $4 \mathrm{mal}$ größer ist: Bei der gleichen Acetonvorlage ist die Extinktion des Disalicylacetons 1,24 und die des Divanillalacetons 0,34. - Auch die Leerwerte verändern sich mit der Zeit, die der Vanillin-KOH-MethanolLösung am meisten, die der Vanillin-KOH-Lösung ohne Methanol am geringsten, nämlich praktisch nicht in den ersten $28 \mathrm{Stdn}$. und später nur minimal. Die Salicylaldehyd-KOH-Lösung steht in der Mitte. Liest man nach 3,5 Stdn. oder nach 12 Stdn. ab, dann ist die Veränderlichkeit der Salicylaldehyd-KOH-Lösung zu diesem Zeitpunkt klein, während die der Vanillin-KOHMethanol-Lösung beträchtlich ist.

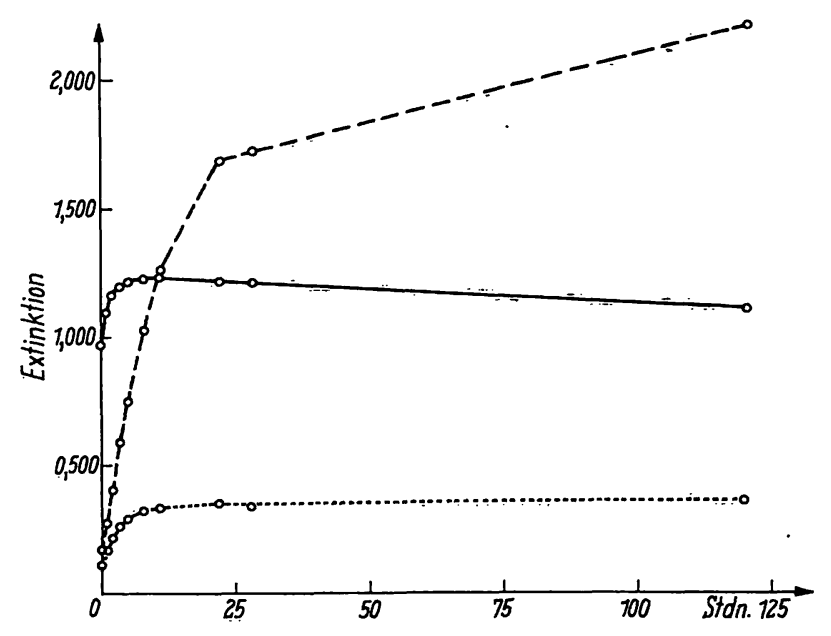

Abb. 3

Verlauf der Extinktion beim Temperaturwechsel von $35^{\circ}$ auf Zimmertemperatur. Divanillalaceton in wäßriger $(. . . .$.$) und$ in methanolhaltiger (-. . - ) Lösung und Disalicylaceton $(-)$

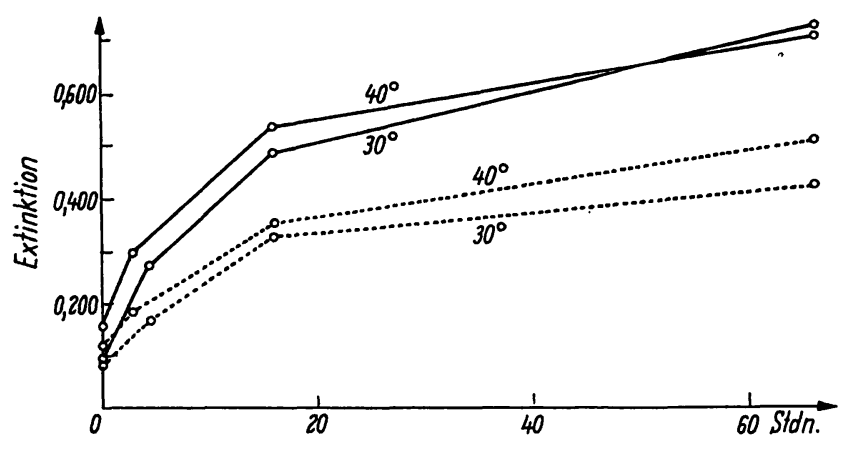

Abb. 4

Einfluß der Reaktionstemperatur auf die Extinktion des Divanillalacetons in methanolhaltiger Lösung ( $\longrightarrow$ ) und des Leerwertes $(\ldots \ldots)$. Bei 0 Std. wird nach vorangegangener 2-stdg. Erwärmung auf $30^{\circ}$ resp. $40^{\circ}$ auf Zimmertemperatur abgekühlt

\section{Die Reaktionstemperatur}

Die Reaktionen des Acetons mit Vanillin oder mit Salicylaldehyd werden bei Temperaturerhöhungen beschleunigt. BAHNER (4) hatte festgestellt, daß die Reaktion des Acetons mit Salicylaldehyd bei seinen gewählten Versuchsbedingungen bei $40^{\circ}$ in $80 \mathrm{Min}$. beendet ist, dagegen bei Zimmertemperatur $10 \mathrm{Stdn}$. dauert; die Endfarbe des Disalicylacetons nach Ausgleich der Temperatureinflüsse war aber weitgehend unabhängig von der Reaktionstemperatur.

Beim Divanillalaceton liegen die Verhältnisse ungünstiger, wenn man unter Methanolzusatz arbeitet. Die Abbildung 4 zeigt, daß die Endfarbe des Divanillalacetons verschieden ist, wenn man die Kühlkappe des Mikrokonvektionsapparates $2 \mathrm{Stdn}$. bei $30^{\circ}$ oder bei $40^{\circ}$ hält. Je höher die Reaktionstemperatur ist, desto höher ist die endgültige Extinktion innerhalb der ersten 24 Stdn. Dies dürfte damit zusammenhängen, daß Divanillalacetonlösungen bei Gegenwart von Methanol keine konstante Farbe erreichen, sondern im Gegensatz zu Disalicylacetonlösungen oder zu Divanillalacetonlösungen ohne Methanolzusatz mit der Zeit dunkler werden. In Methanol laufen Sekundärreaktionen $a b$, die bei hoher Reaktionstemperatur beschleunigt werden, so da $\beta$ die Temperatur die Endfarbe beeinflussen kann. Auch die Reaktionszeit, also die Dauer des Erwärmens der Mikrokonvektionsapparate, beeinflußt daher die Endfarbe. Bei der alkalischen Vanillinlösung ohne Methanolzusatz haben wir auf eine genauere Bestimmung der Reaktionsgeschwindigkeit verzichtet, weil die Vanillinmethode aus den im vorigen Kapitel aufgezeigten Gründen nicht mit der Salicylaldehydmethode konkurrieren kann.

\section{Diskussion}

Vanillin läßt sich auf zweierlei Weise in alkalischer Lösung zur Acetonbestimmung verwenden, nämlich in wäßriger alkalischer Lösung oder in alkalischer Lösung unter Zusatz vor Methanol. - Die wäßrige Vanillinlösung ist heller und beständiger als eine Lösung mit einem Methanolzusatz oder als eine wäßrige alkalische Salicylaldehydlösung. Aber auch wäßrige Vanillinlösungen werden im Licht heller. Die Unterschiede der Extinktionen bei unterschiedlicher Beleuchtung und Atmosphäre sind geringer als beim Salicylaldehyd, sie 
stören aber wie bei diesem die quantitative Acetonbestimmung, denn je nach den Lichtverhältnissen oder der Luftmenge im Apparat können die Werte beträchtlich schwanken. Wie bei der Bestimmung des Acetons mit Salicylaldehyd müssen daher Tageslicht und Luft vom Vanillin-KOH-Reagenz ferngehalten werden, indem man bei künstlicher Beleuchtung und in Stickstoffatmosphäre arbeitet. Die Löslichkeit des Vanillins in Wasser und in Alkali ist aber zu klein, als daß damit die Extinktionen erreicht werden können, die man mit methanolhaltiger Vanillinlösung oder mit einer wäßrigen Salicylaldehydlösung erzielen kann. In $0,1 \mathrm{~m} l$ Blut lassen sich daher die physiologischerweise vorkommenden Acetessigsäurekonzentrationen nicht mit einer wäßrigen, alkalischen Vanillinlösung bestimmen. Mit einer entsprechend größeren Blutmenge ist die Mikrokonvektionsmethode von BAHNER nicht mehr ohne weiteres durchführbar.

Vanillin ist in Methanol sehr gut löslich (bis 50\%). Methanol ermöglicht auch höhere $\mathrm{KOH}-$ Konzentrationen, die wegen der Farbvertiefung erwünscht sind, aber es hat verschiedene Nacbteile:

1. Methanol schwächt in höheren Konzentrationen die Farbe des Divanillalacetons ab (Abb. 2);

2. die Extinktionen des Divanillalacetons hängen stark vom Methanolgehalt $\mathrm{ab}$, so daß Schwankungen im Methanolzusatz und Änderungen in der Methanolkonzentration während des Erwärmens einen bedeutenden Einfluß auf die Endfarbe haben (Abb. 2);

3. bei Gegenwart von Methanol strebt die Extinktion des Divanillalacetons keinem konstanten Endwert zu, sondern steigt stetig an (Abb. 3);

4. bei Methanolzusatz hängt die Endfarbe in erhöhtem Maße von der Reaktionstemperatur ab (Abb. 4).

Der Methanolzusatz stört die Reaktion des Acetons mit Vanillin so sehr, daß sie sich zur quantitativen Acetonbestimmung nicht eignet. Der Meinung von LINDBERG und ÖRSTRöM (7), daß die Methoden mit Salicylaldehyd und mit Vanillin gleich gut seien und sehr gut übereinstimmende Werte ergäben, müssen wir widersprechen. Diese Autoren haben nicht bei künstlicher Beleuchtung und nicht in Stickstoffatmosphäre gearbeitet und sie haben die langsame Einstellgeschwindigkeit des Gleichgewichts des Divanillalacetons und des Disalicylacetons beim Temperaturwechsel nicht beachtet.

Vanillin eignet sich zur Acetonbestimmung also nicht gleich gut wie Salicylaldehyd. Bei den günstigsten Vanillin- und KOH-Konzentrationen sind ohne Methanolzusatz die Extinktionen fast $4 \mathrm{mal}$ niedriger als mit der Salicylaldehydmethode. Unter Methanolzusatz laufen Sekundärreaktionen $\mathrm{ab}$, die die quantitative Bestimmung sehr stören.

\title{
Literatur
}

1. Nikırın, E. K., Žur. Obščej. Chim. 8, 2264 (1937). - 2. BäckSTRöm, H. L. J., Z. analyt. Chem. 123, 96 (1942). - 3. Levine, V. E. und M. Taterka, Clin. Chem. (New York) 3, 646 (1957). 4. BAHNER, F., Biochem. Z. 323, 327 (1952). - 5. BAHNER, F.,
Biochem. Z. 323, 318 (1952). - 6. Barner, F., Chemie-Ing.Techn. 25, 89 (1953). - 7. LindBerg, O. und A. Örström, Ark. Kem., Mineralog. Geol. Ser. A, 18/3, 1 (1945).

Professor Dr. med. F. Bahner

Endokrinolog. Abt. der Med. Poliklinik der Universität 69 Heidelberg, Hospitalstr. 3

\section{Mikropräparative Trennung der Lipidklassen von Milch und Blutserum}

\author{
Von G. CZEGLÉdi-Jankó \\ Aus dem Institut für Ernäbrungswissenschaft, Budapest, Ungarn (Direktor: Prof. Dr. R. Tarjän)
}

(Der Schriftleitung zugegangen am 16. März 1964)

\begin{abstract}
Zur präparativen Trennung der Hauptlipidklassen, in erster Linie in Milch und Blutserum wutde eine manuell gegossene, „dicke“ Kieselgelschicht angewendet. Mit dieser Methode konnten die einzelnen Hauptklassen in kurzer Zeit in solchen Mengen erhalten werden, daß die Fettsäurezusammensetzung selbst der Cholesterinesterfraktion der Milch mit einem katherometrischen Gaschromatographen ermittelt werden konnte.

Das qualitative Bild der getrennten Lipide und die Bestimmung der Fettsäurezusammensetzung der Lipidklassen lieferten einige neue Angaben hinsichtlich der Unterschiede zwischen Frauenmilch und Kuhmilch.

The preparative separation of the main classes of lipids, particularly in milk and blood serum, was performed on manually prepared "thick" layers of silica gel. The main lipid fractions of milk were obtained quickly and in sufficient quantities for a determination of the fatty acid composition, even in the cholesterol ester fraction, with a catherometric gas chromatograph.

The qualitative picture of the separated lipids and the establishment of the faity acid composition of the lipid classes yielded new data on the differences between human and cow's milk.
\end{abstract}

Für die klinische Diagnostik sind sowohl die Kenntnis der Zusammensetzung der Lipide als auch die Methoden zur Bestimmung der Komponenten von immer größerem Interesse. In einer seiner Mitteilungen betont SCHRADE
(1) die klinische Bedeutung der Trennung von Blutserumlipiden nach Verbindungsklassen und der Bestimmung der Fettsäurekomponenten in den einzelnen Lipidfraktionen. 